\title{
Is Endoscopy Necessary for the Measurement of Disease Activity in Ulcerative Colitis?
}

Peter D.R. Higgins, M.D., Ph.D., Marc Schwartz, M.D., John Mapili, M.D., and Ellen M. Zimmermann, M.D. Division of Gastroenterology, University of Michigan, Ann Arbor, Michigan

OBJECTIVES: Many disease activity indices are used to measure ulcerative colitis. Invasive indices incorporate an endoscopic score, while noninvasive indices do not require endoscopy. In clinical practice, many patients are treated based on their symptoms without endoscopic evaluation. However, invasive indices are commonly used in clinical research. Our objective was to determine whether endoscopy is necessary for the assessment of disease activity in patients with ulcerative colitis.

METHODS: $\quad$ Sixty-six consecutive ulcerative colitis patients were evaluated with invasive indices: the St. Mark's index and the Ulcerative Colitis Disease Activity Index (UCDAI); and noninvasive indices: the Simple Clinical Colitis Activity Index (SCCAI) and the Seo index. The correlations between the indices were measured. The contribution of the endoscopic items was measured with linear regression modeling. The overlap of endoscopy with other items in the standard indices was determined through factor analysis.

RESULTS: $\quad$ The two noninvasive indices correlated well with the invasive St. Mark's Index (SCCAI 0.86, Seo 0.70). After adjusting for the three noninvasive UCDAI items, the UCDAl endoscopy item predicted only $0.04 \%$ of the variance in the St. Mark's index. Factor analysis demonstrated that this is because the endoscopy items in the invasive indices correlate with stool frequency and stool blood items.

CONCLUSIONS: Endoscopy items contribute little additional information to indices of disease activity in ulcerative colitis. The clinical practice of treating patients based on reported symptoms is appropriate. The use of noninvasive indices in clinical trials could lower study costs and may increase subjects' willingness to participate.

(Am J Gastroenterol 2005;100:355-361)

\section{INTRODUCTION}

Multiple disease activity indices for ulcerative colitis have been devised, but none have been rigorously validated. Most clinical trials have used multiple disease activity indices since there is no clear gold standard. Patients in clinical trials are often subjected to multiple endoscopies for repeated evaluation of disease activity because endoscopy is part of most of the standard indices, including the St. Mark's index and the Ulcerative Colitis Disease Activity Index (UCDAI). However, in clinical practice, patients with known ulcerative colitis who experience typical symptoms (stool urgency, frequency, and stool blood) of a disease flare are often treated empirically without endoscopic confirmation. The expense and discomfort of endoscopy do increase the cost and may decrease recruitment for clinical trials. It is not clear that endoscopy is necessary for the measurement of disease activity in a patient with an established diagnosis of ulcerative colitis.

The measurement of disease activity in ulcerative colitis is critical in determining whether new therapies are effective, but there is no gold standard for measuring disease activity in ulcerative colitis. The first published attempt at quantifying disease activity was part of the first trial of corticosteroids by
Truelove and Witts, who defined mild, moderate, and severe colitis (1) in 1955, based on symptoms, signs, and physician assessment. They did not validate their measures, and did not include a category for remission, since they did not have an effective remission-inducing therapy prior to their clinical trial. In 1978, these broad definitions were transformed into a continuous point scale by Powell-Tuck et al., commonly called the St. Mark's index (aka Powell-Tuck index) (2). The St. Mark's index adds endoscopic findings to physical examination, temperature, and eight other assessment components, making it somewhat cumbersome and difficult to apply clinically. The St. Mark's index is widely used but has never been validated.

To streamline the assessment of disease activity for clinical trials, Sutherland developed the Ulcerative Colitis Disease Activity Index (UCDAI) (3), a simplified index with four components, including physician assessment and endoscopy. A similar approach was used by Schroeder et al. to develop the Mayo score (4), which has four very similar components including endoscopy. Neither of these indices has been validated, but since they are simpler than the St. Mark's index to perform, they have come into common use in clinical trials. However, with repeated assessment required in clinical trials, 
the requirement for endoscopy can become burdensome in both patient discomfort and costs.

Two groups have therefore developed nonendoscopic activity indices. Seo et al. used regression modeling to develop an index based on symptoms and hemoglobin, albumin, and Westergren erythrocyte sedimentation rate (5). This index was developed by model fitting to Truelove and Witts' ordinal categories of mild, moderate, and severe disease. Seo has shown that elevated scores on this index correlate with endoscopic activity (6) and with future colectomy (7) in retrospective analyses in Japanese patients. Walmsley et al. (8), developed the Simple Clinical Colitis Activity Index (SCCAI), a survey of six questions about symptoms, and showed that it correlated well with the St. Mark's index, the Seo index, and with a validated quality-of-life measure, the Inflammatory Bowel Disease Quality-of-Life index (IBDQ), in British patients. Neither of these indices has come into widespread use in the United States, and neither has been formally validated.

In the absence of a validated gold standard for disease activity, experts in the field have advocated different indices, which has led individuals performing clinical trials to measure multiple indices in their subjects $(9,10)$. Using multiple indices protects authors from post hoc criticism from reviewers and regulatory agencies, but is expensive and onerous for patients.

The only validated index in the field is a quality-of-life index. The IBDQ has repeatedly been validated in multiple settings, and has become the de facto gold standard for quality of life in ulcerative colitis. This absence of a gold standard for disease activity has led authors (i.e., Walmsley, Seo) to show that their disease activity indices correlate with the IBDQ. These correlations do support the face validity of the disease activity indices, but do not substitute for formal validation.

In this study, we aim to determine the necessity of endoscopy for the assessment of disease activity by (i) measuring the correlation of the noninvasive indices with the classic invasive index, the St. Mark's index, (ii) measuring the contribution of endoscopy to the variance of the St. Mark's index by determining how much the endoscopic information adds to the predictiveness of a linear regression model of the St. Mark's index using the components of the UCDAI, and (iii) determining the overlap of endoscopy with other items in the standard indices through factor analysis of all twenty-six items in the four activity indices.

\section{METHODS}

\section{Patients}

Seventy-four consecutive patients were identified by searching the endoscopy schedule at the University of Michigan Medical Procedures Unit for patients with ulcerative colitis who were scheduled for lower endoscopy. The University of Michigan is a regional referral center with a large local primary care base and more than 1,300 outpatient gastroenterol- ogy clinic visits for ulcerative colitis in 2002. The population from which our study sample was derived tend toward active disease, and is likely more representative of patients participating in clinical trials than a community-based population. Two patients were nonnative speakers (from Mexico and Korea), and could not read and understand the consent form. Two patients refused to participate, stating that they were too nervous about their procedure to complete surveys. The remaining 70 patients consented to participate in the study. Two patients were excluded from data analysis because of incomplete data (random failure to collect all laboratory tests for Seo index). Two additional patients were excluded from data analysis after consent was obtained because of confounding medical illness: one patient was found to have ischemic colitis and a second patient was excluded for concurrent chemotherapy for metastatic breast cancer.

\section{Patient Evaluation}

Before the scheduled endoscopy and sedation, informed consent was obtained, and survey forms to collect data for the SCCAI, Seo index, UCDAI, and St. Mark's index, and IBDQ (total of 50 questions) were administered. Blood samples for hemoglobin, albumin, and Westergren sedimentation rate for the Seo index were drawn before endoscopy. Primary gastroenterologists or endoscopists were asked to assess disease severity before endoscopy for the UCDAI. After the endoscopy, endoscopists (15 total endoscopists) were asked to assess the endoscopic appearance for the UCDAI and St. Mark's indices for their own patients.

\section{Statistical Analysis}

Because many of the patients were undergoing colonoscopy for surveillance of longstanding pancolitis rather than active disease, this sample of patients is overrepresented in low disease activity levels (right skewed). Therefore, both the nonparametric Spearman correlations and the Pearson correlation with transformed values were performed. Index values were mathematically transformed to better approximate normal distributions for the Pearson correlation. Transformed variables with a skewness closest to zero and normal distributions as confirmed by inspection of q-q plots were used. These transformations included the square root transformation (selected with the ladder function in Stata) for the St. Mark's index, the UCDAI, and the SCCAI, and a log transformation [ $\ln ($ Seo-62.77) that has a skewness of zero, selected with the lnskew0 function in Stata] for the Seo index. The sample size was selected to achieve a $95 \%$ confidence interval for the Pearson correlation of width less than 0.20 . Based on Walmsley's data (8), we estimated the $\rho$ for the correlation between the SCCAI and the St. Mark's Index at 0.8, which required $\mathrm{N}$ of 53 for the desired level of precision, and we added 10 for a conservative estimate of 63 patients. The $95 \%$ confidence limits $(\rho 1, \rho 2)$ for the Pearson correlations were calculated using the arcsin square root transformation with the formulas: $\rho 1=(\exp (2 * \mathrm{z} 1)-1) /(\exp (2 * \mathrm{z} 1)+1)$ and $\rho 2=(\exp (2 * \mathrm{z} 2)-1) /(\exp (2 * \mathrm{z} 2)+1)$, where 
$\mathrm{z}=0.5 \ln \{(1+\mathrm{r}) /(1-\mathrm{r})\}, \mathrm{z} 1=\mathrm{z}-1.96 / \mathrm{sqrt}(\mathrm{n})$, and $\mathrm{z} 2=$ $z+1.96 /$ sqrt(n) in Microsoft Excel (Microsoft, Bellevue, WA).

The determination of the contribution of endoscopy to the predictiveness of invasive activity indices was performed by doing linear regression modeling, using the St. Mark's score as the dependent variable, and the items of the UCDAI as independent variables. The two models, one with and one without the endoscopic score item, were compared to determine the independent contribution of endoscopy to the predictiveness of the model. Each model was evaluated for the presence of outliers, linearity, and heteroskedasticity through inspection of scatterplots and residual plots.

The segregation of the items from the four disease activity indices into inherent factors was analyzed with factor analysis with orthogonal rotation. The first four factors had eigenvalues greater than one, and these factors were retained for further analysis. Items were grouped by their predominant factor loading and the role of endoscopic items was interpreted. Three of the 26 items measured are dichotomous variables (i.e., anorexia). The multivariate normality of the factor analysis was expected to be sufficiently robust to tolerate these few dichotomous variables (11). In addition, we ran the factor analysis without these three dichotomous variables, and no significant changes in the factors or factor loading were seen (data not shown). All statistical calculations (with the exception of the Pearson confidence limits) were done with Stata 8.2 (Stata, College Station, TX).

\section{RESULTS}

A representative sample of patients undergoing lower endoscopy for ulcerative colitis was obtained at our institution by approaching 74 consecutive patients scheduled for lower endoscopy with a diagnosis of ulcerative colitis. This sample included both very ill inpatients and healthy outpatients undergoing surveillance for colonic dysplasia. The participation rate was $94.6 \%$. The participants are described in Table 1 . The distributions of disease activity values for each

Table 1. Characteristics of Patient Sample

\begin{tabular}{lcc}
\hline Characteristics & $\begin{array}{c}\text { Mean } \pm \text { Standard } \\
\text { Deviation and Percentage }\end{array}$ & Range \\
\hline Age & $40.5 \pm 15.2$ yr & $18-90$ \\
St. Mark's index & $5.28 \pm 4.23$ points & $0-17$ \\
UCDAI & $4.64 \pm 3.94$ points & $0-12$ \\
SCCAI & $4.00 \pm 3.84$ points & $0-16$ \\
Seo index & $141.07 \pm 52.42$ points & $75.4-263.2$ \\
IBDQ & $179.21 \pm 48.00$ points & $59-224$ \\
Female & $54.6 \%$ & \\
Caucasian & $89.4 \%$ & \\
African American & $6.1 \%$ & \\
Oral 5-ASA use & $77.3 \%$ & \\
Rectal therapy use & $11.2 \%$ & \\
Steroid use & $25.7 \%$ & \\
Thiopurine use & $21.2 \%$ & \\
\hline
\end{tabular}

of the activity indices (all normalized to a range of 0-100 for comparison) are presented in Figure 1. A wide range of disease activity was present in the study sample, and scores near the minimum and maximum for each measure were represented in this cohort. Because correlation coefficients are used here to measure strength of a linear association, we first assessed the linearity of these relationships. Those variables that had nonlinear relationships were mathematically transformed to approximate a normal distribution, and the relationships after transformation were linear.

\section{Noninvasive Indices Correlated Well with Invasive Indices without the Benefit of Endoscopic Information}

The noninvasive indices were evaluated to determine if they correlated well with the original invasive index, the St. Mark's index. In order to have a benchmark for correlation and variability, the correlation of the invasive UCDAI with the St. Mark's index was measured. The Pearson correlation of the square root transformed values for the St. Mark's index and the square root transformed UCDAI was 0.881 (95\% CI $0.814-0.925)$.

The Pearson correlations of the square root transformed St. Mark's index and the noninvasive indices, the square root transformed SCCAI, and the log-transformed Seo index, were 0.908 (95\% CI 0.855-0.942) and 0.803 (95\% CI 0.6990.873 ), respectively. The noninvasive SCCAI and Seo indices were nearly as well correlated with the St. Mark's index as the UCDAI, and the SCCAI had a better correlation than the Seo index. This finding was confirmed with the nonparametric Spearman correlations of the untransformed indices. The

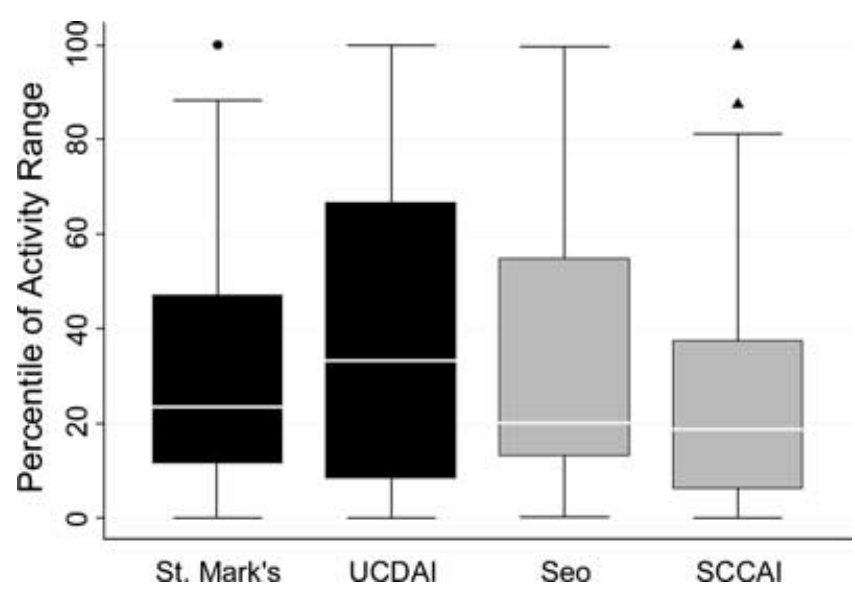

Figure 1. A broad range of disease activity levels were included, and disease activity values were skewed toward high activity. Disease activity index values were normalized to a $0-100$ scale for comparison. Black boxes indicate the invasive (endoscopic) indices, while gray boxes indicate the noninvasive indices. For all indices low scores represent less disease activity. The interquartile range (IQR) is indicated by the shaded rectangle, the median is indicated by a horizontal line, and the tails and whiskers indicate the median \pm 2 IQR. Outlying values more than 2 IQR from the median are identified by individual points. 
Spearman correlation of the St. Mark's index with the UCDAI was 0.867 , the correlation with the SCCAI 0.866, and the correlation with the Seo index was 0.705. Again, the SCCAI correlated nearly as well with the St. Mark's index as did the invasive UCDAI, and the Seo index correlated well, but not as well as the SCCAI. The noninvasive indices correlated well with the St. Mark's index without the benefit of endoscopic information.

\section{Noninvasive Indices also Correlated with St. Mark's Index in Patients with Active Disease}

While this cohort had a full range of disease severity, it contained many patients in remission. Patients in clinical treatment trials will not necessarily be in remission, and it is important to demonstrate that this correlation is valid for the subset of patients with active disease, who are more likely to enroll in clinical treatment trials. The Spearman correlations were reexamined using only subjects with a St. Mark's index of $>3(n=40)$. The correlation of the St. Mark's index with the UCDAI changed only from 0.8666 to 0.8661 . Similarly, the correlation of the St. Mark's index with the SCCAI changed only slightly from 0.8660 to 0.8683 , and the correlation with the Seo index increased slightly from 0.7050 to 0.7220 . The Spearman correlations with the UCDAI were also reexamined using only subjects with a UCDAI of $>4$ $(\mathrm{n}=31)$. The correlation of the UCDAI with the St. Mark's index was slightly reduced from 0.8666 to 0.7933 . Similarly, the correlation with the SCCAI decreased from 0.8517 to 0.8290 , and the correlation with the Seo index changed from 0.7843 to 0.7311 . The noninvasive indices continued to correlate well with the invasive St. Mark's index in the subset of patients with active disease. This is presented graphically in the correlation matrix scatter plot of untransformed values of the indices in Figure 2, which divides subjects into mild, moderate, and severe disease as defined by Truelove and Witts, using the cutoffs determined by Seo.

\section{The Endoscopic Items in Invasive Indices Added Little to the Measurement of Disease Activity}

In order to determine how much the endoscopic component of invasive indices contributes to the measurement of disease activity, the components of the UCDAI were used to predict the St. Mark's index with linear regression. The four components of the UCDAI predicted the St. Mark's index well, with $\mathrm{R}^{2}=0.7980$. The contribution of the endoscopic item to this prediction was then determined by removing it from the model. After removal of the endoscopic item from the model, the remaining three items also predicted the St. Mark's index well, with $\mathrm{R}^{2}=0.7976$, demonstrating that the UCDAI endoscopy item predicted only $0.04 \%$ of the variance in the St. Mark's index beyond the variability explained the by noninvasive components.

It is possible that the UCDAI endoscopic item was uninformative because the endoscopic item used in the UCDAI is a poor measure, since it has never been validated. A similar analysis was performed in reverse to determine whether the

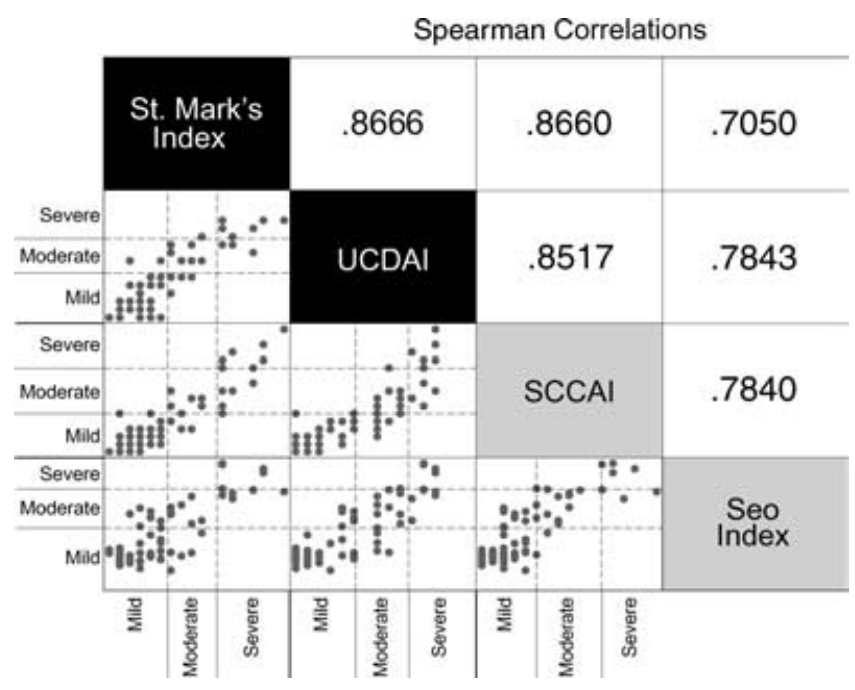

Figure 2. A matrix of scatter plots and Spearman correlations of all four clinical disease activity indices, divided into Truelove and Witt's mild, moderate, and severe categories. The four clinical disease activity indices are presented as a matrix. The invasive (endoscopic) indices are indicated by black boxes, and the noninvasive indices are indicated by gray boxes. At the lower left, at the intersection of each pair of indices, a scatter plot of the two indices is presented, with dashed lines marking the delineation between mild, moderate, and severe disease. These cutoffs were determined for the Seo index to be 150 (between mild and moderate) and 220 (between moderate and severe) by Seo. Linear regression using the data in this study showed that these cutoffs are equivalent to 6 and 10.6 in the St. Mark's Index, 5.2 and 9.8 in the UCDAI, and 5 and 11 in the SCCAI. At the upper right, at the intersection of each pair of indices, the Pearson correlation for that pair is presented.

endoscopic item in the St. Mark's index might be more informative. All of the individual items in the St. Mark's index were used to predict the disease activity as measured by the UCDAI. The $\mathrm{R}^{2}$ for prediction of the UCDAI with all of the items in the St. Mark's index was 0.9102 . The $\mathrm{R}^{2}$ decreased to 0.8765 without the endoscopic item in the model, showing that the St. Mark's endoscopic item contributed only 3.37\% to the variability in the UCDAI. Examination of the subjects with active disease confirmed this finding in this subset of patients, as the UCDAI items predicted the St. Mark's index with a $\mathrm{R}^{2}$ of 0.7106 , and this decreased minimally to 0.7088 $(0.18 \%)$ without the endoscopic item. St. Mark's index items predicted the UCDAI score with an $\mathrm{R}^{2}$ of 0.8680 , and this decreased minimally to $0.8548(1.3 \%)$ without the endoscopic item. Our data show that the current endoscopic items in standard invasive indices contribute very little $(0.04-3.37 \%)$ to the variability in measurement of disease activity.

\section{The Endoscopic Items Correlate with Disease Activity}

One possible explanation for our finding that current endoscopic items contribute little to the measurement of disease activity is that the endoscopic items themselves are flawed or inaccurately assess endoscopic activity. To test this possibility, we evaluated whether the endoscopic items, which have never been validated, correlate with measures of disease 
activity, using the St. Mark's index and the UCDAI as our standard disease activity measures. These endoscopic items are both simple ratings of friability, which may not be an accurate measure of endoscopic disease activity. We used the endoscopic item from each index (Table 2) to test correlation with the other disease activity index (rather than with its own overall index), as we would expect each endoscopic item to correlate well with the overall index of which it is a component.

The endoscopic item from the St. Mark's index correlated well with the disease activity as measured by the UCDAI, with a Spearman rank correlation of 0.775 . The endoscopic item from the UCDAI also correlated well with disease activity as measured by the St. Mark's index, with a Spearman rank correlation of 0.720 . The lack of informativeness of the endoscopic items was not largely due to failure of the items to correlate with disease activity. The endoscopic items correlated well with measures of disease activity, but they added little to the information from other items in the indices.

\section{Endoscopic Items Segregated with Stool}

\section{Frequency/Urgency and Stool Blood in Factor Analysis}

Another explanation for the lack of additional information from endoscopic items is that the inherent factors that are measured by endoscopy are already measured by other items in the activity indices. Items in any disease activity scale measure some aspect of unidentified inherent factors in the disease itself. It is not uncommon for disease activity items to be measuring the same inherent factor. The endoscopic items in the invasive indices could be measuring the same inherent factor as noninvasive questions. If this were the case, endoscopic information would be redundant.

To test the hypothesis that the inherent factors measured by endoscopy have already been measured by noninvasive items, the inherent factors measured by the items in all four activity indices were determined by subjecting the 26 items from all four activity indices to factor analysis with orthogonal rotation to maximize the independence of factors. Four factors were found to have eigenvalues of greater than one, and were retained for analysis. The scree plot is presented in Figure 3. These four inherent factors were responsible for $86.06 \%$ of the variance of the 26 items.

Table 2. Endoscopic Items from the St. Mark's Index and the UCDAI

\begin{tabular}{ll}
\hline $\begin{array}{l}\text { Disease Activity } \\
\text { Index }\end{array}$ & $\begin{array}{l}\text { Endoscopic } \\
\text { Items }\end{array}$ \\
\hline St. Mark's Index & $\begin{array}{l}\text { In the endoscopist's opinion, is the mucosa: } \\
\text { (i) Nonhemorrhagic } \\
\text { (ii) Friable } \\
\text { (iii) Has spontaneous bleeding } \\
\text { In the endoscopist's opinion, is the mucosa: } \\
\text { (i) Normal } \\
\text { (ii) Mildly friable } \\
\text { (iii) Moderately friable } \\
\text { (iv) Exudative with spontaneous bleeding }\end{array}$ \\
\end{tabular}

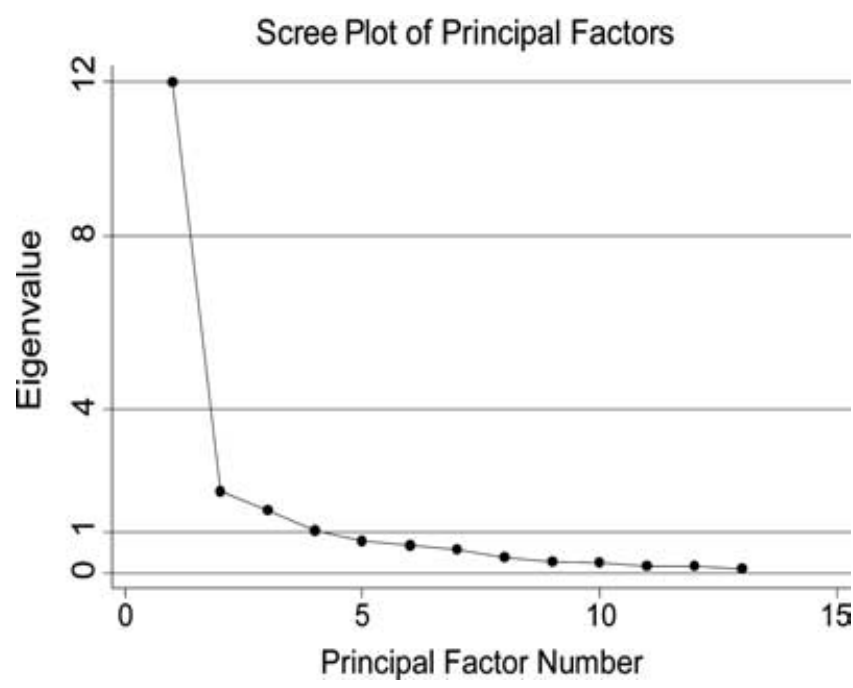

Figure 3. Scree plot of eigenvalues for inherent factor analysis of items in disease activity indices. The first four inherent factors have eigenvalues greater than one. These four inherent factors were retained for further analysis. These factors are presented in Table 3, in which each inherent factor is given a name based on the identities of the items that segregated to that factor.

The rotated factor loadings of the 26 items for each factor and their uniqueness are presented in Table 3. Inherent factor 1 was related to stool frequency and urgency, while inherent factor 2 was related to stool blood. Inherent factor 3 captured extraintestinal manifestations and abdominal symptoms, and inherent factor 4 was related to the systemic laboratory manifestations of disease. The physician rating item was distributed between inherent factors 1 and 2 . The temperature item from the St. Mark's index had a high uniqueness coefficient, and was likely measuring a distinct inherent factor.

It is notable that the endoscopic items did not measure a distinct inherent factor, and had little uniqueness $(0.23$ for the UCDAI endoscopic item and 0.36 for the St. Mark's index endoscopic item). In fact, the variance of these two items is distributed between the inherent factors 1 (stool frequency/urgency) and 2 (stool blood). While the endoscopic items did correlate with disease activity, the other items in the disease activity indices that measure stool frequency/urgency and stool blood made the additional information gained from the endoscopic items redundant.

\section{DISCUSSION}

We have found that the current endoscopic items in standard invasive indices actually contribute very little to the variability in measurement of disease activity. One explanation of this is that endoscopy itself is not an accurate measure of disease activity. However, clinical experience and the nature of the disease process of mucosal inflammation suggest that endoscopic findings should strongly correlate with 
Table 3. Results of Factor Analysis of All Twenty-Six Items in the St. Mark's Index, the UCDAI, the SCCAI, and the Seo Index

\begin{tabular}{|c|c|c|c|c|c|}
\hline Inherent Factor Name & Frequency/Urgency & Stool Blood & Extraintestinal & Labs & Uniqueness \\
\hline Inherent factor number & 1 & 2 & 3 & 4 & \\
\hline$\%$ of variance predicted & $62.2 \%$ & $10.4 \%$ & $8.0 \%$ & $5.5 \%$ & \\
\hline \multicolumn{6}{|l|}{ Items } \\
\hline $\begin{array}{l}\text { SCCAI day BM } \\
\text { Seo frequency } \\
\text { UCDAI frequency } \\
\text { SM frequency } \\
\text { SCCAI night BM } \\
\text { SCCAI urgency } \\
\text { SM consistency } \\
\text { SCCAI well being } \\
\text { SM general health } \\
\text { SM pain }\end{array}$ & $\begin{array}{l}0.85 \\
0.84 \\
0.83 \\
0.82 \\
0.71 \\
0.64 \\
0.60 \\
0.57 \\
0.56 \\
0.55\end{array}$ & $\begin{array}{l}-0.33 \\
-0.40 \\
-0.29 \\
-0.32 \\
-0.29 \\
-0.19 \\
-0.21 \\
-0.31 \\
-0.37 \\
-0.28\end{array}$ & $\begin{array}{r}0.05 \\
-0.05 \\
0.04 \\
0.01 \\
-0.05 \\
0.21 \\
0.12 \\
0.38 \\
0.29 \\
0.34\end{array}$ & $\begin{array}{r}-0.16 \\
-0.16 \\
-0.10 \\
-0.10 \\
-0.42 \\
-0.37 \\
-0.31 \\
-0.28 \\
-0.38 \\
0.03\end{array}$ & $\begin{array}{l}0.14 \\
0.10 \\
0.21 \\
0.21 \\
0.23 \\
0.38 \\
0.48 \\
0.37 \\
0.31 \\
0.50\end{array}$ \\
\hline $\begin{array}{l}\text { SCCAI blood } \\
\text { UCDAI blood } \\
\text { Seo blood } \\
\text { SM blood }\end{array}$ & $\begin{array}{l}0.27 \\
0.30 \\
0.15 \\
0.31\end{array}$ & $\begin{array}{l}-0.93 \\
-0.91 \\
-0.91 \\
-0.88\end{array}$ & $\begin{array}{l}0.07 \\
0.06 \\
0.04 \\
0.10\end{array}$ & $\begin{array}{l}-0.09 \\
-0.14 \\
-0.13 \\
-0.12\end{array}$ & $\begin{array}{l}0.04 \\
0.06 \\
0.13 \\
0.10\end{array}$ \\
\hline $\begin{array}{l}\text { UCDAI endoscopy } \\
\text { SM endoscopy } \\
\text { UCDAI Dr. rating }\end{array}$ & $\begin{array}{l}0.49 \\
0.46 \\
0.55\end{array}$ & $\begin{array}{l}-0.67 \\
-0.61 \\
-0.61\end{array}$ & $\begin{array}{r}-0.22 \\
-0.23 \\
0.10\end{array}$ & $\begin{array}{l}-0.18 \\
-0.03 \\
-0.26\end{array}$ & $\begin{array}{l}0.23 \\
0.36 \\
0.25\end{array}$ \\
\hline $\begin{array}{l}\text { SM extraintestinal } \\
\text { SM anorexia } \\
\text { SM nausea vomiting } \\
\text { SM abd tenderness } \\
\text { SCCAI extraintestinal }\end{array}$ & $\begin{array}{r}-0.02 \\
0.18 \\
-0.05 \\
0.38 \\
-0.16\end{array}$ & $\begin{array}{r}0.09 \\
-0.33 \\
-0.04 \\
-0.24 \\
0.18\end{array}$ & $\begin{array}{l}0.55 \\
0.55 \\
0.46 \\
0.42 \\
0.42\end{array}$ & $\begin{array}{r}-0.14 \\
-0.11 \\
-0.04 \\
-0.06 \\
0.00\end{array}$ & $\begin{array}{l}0.67 \\
0.55 \\
0.78 \\
0.61 \\
0.76\end{array}$ \\
\hline $\begin{array}{l}\text { Seo Westergren ESR } \\
\text { Seo albumin } \\
\text { Seo hemoglobin }\end{array}$ & $\begin{array}{r}0.35 \\
-0.34 \\
-0.24\end{array}$ & $\begin{array}{r}-0.15 \\
0.38 \\
0.39\end{array}$ & $\begin{array}{r}0.03 \\
-0.08 \\
-0.08\end{array}$ & $\begin{array}{r}-0.77 \\
0.63 \\
0.61\end{array}$ & $\begin{array}{l}0.26 \\
0.34 \\
0.41\end{array}$ \\
\hline SM temperature & 0.05 & -0.04 & -0.03 & 0.20 & 0.95 \\
\hline
\end{tabular}

disease activity. A second explanation is that the particular endoscopic items from each invasive index are not good measures of endoscopic activity. This seems possible since the items themselves are subjective and have never been validated. However, our data show that the endoscopic items did correlate well with disease activity measures, so that both of these explanations were refuted.

An alternative explanation is that the information derived from endoscopic activity is measuring the same inherent factors in disease activity as were measured by other items in the invasive indices. If this were the case, adding endoscopic information would add little to the measurement of disease activity. Factor analysis supported this explanation, identifying two inherent factors, stool frequency/urgency and stool blood, to which the endoscopic items segregated. The explanation for why endoscopy contributes little to the disease activity indices is that the information captured by standard endoscopic items is already contained in other noninvasive items in standard indices.

This could lead one to argue that only noninvasive indices should be used in clinical trials and in clinical assessment of patients with known ulcerative colitis. This would likely be more cost-efficient and less uncomfortable for patients. In our experience, patients with active colitis find unsedated sigmoidoscopy, which is commonly used in clinical trials, quite uncomfortable. A recent clinical trial in ulcerative colitis (12) used unsedated sigmoidoscopy every 2 wk throughout the trial to assess disease activity. Our data suggest that these endoscopies may not have added significantly to the measurement of disease activity, and they likely added significantly to study costs and subject discomfort.

A counterargument is that endoscopy is necessary in clinical trials for obtaining tissue samples to assess drug delivery or to study cellular mechanisms. However, this purpose could be achieved by comparing biopsies at the beginning and end of the trial, eliminating the need for interim endoscopies with biopsies.

While these data make noninvasive indices attractive, there are critical limitations to current noninvasive indices. Neither the SCCAI nor the Seo indices have been rigorously validated, and neither has been demonstrated to be reproducible nor responsive to change. It is possible that a better endoscopic index that incorporated more precise endoscopic parameters would add new information to current activity indices. Both the St. Mark's endoscopic item and the UCDAI endoscopic item focus solely on friability and bleeding, which along with many other signs of endoscopic activity in ulcerative colitis, 
has been found to have poor interobserver reliability $(\kappa=$ $0.38)(13)$.

While endoscopy may add little to disease activity assessment in the broad range of patients in this sample, endoscopy is valuable in both clinical trials and clinical practice for other reasons. In clinical trials, biopsies obtained at endoscopy can be used to measure tissue drug levels or to study molecular mechanisms of drug actions. In clinical practice, endoscopy is necessary to confirm diagnosis, to evaluate the extent of disease, to evaluate disease that is unresponsive to therapy, and to assess for complications including stricture, dysplasia, or cancer. The histologic examination of biopsies obtained at endoscopy also yields valuable information that can help guide therapy.

Endoscopy items contribute little to standard indices of disease activity in ulcerative colitis. This appears to be largely due to the fact that other items in the invasive indices segregate to the same inherent factors, and therefore endoscopic items add little additional information. Our data support the standard clinical practice of following the self-reported symptoms of patients with ulcerative colitis, rather than reassessment with endoscopy. Disease flares that are unresponsive to standard therapy should be evaluated with endoscopy. However, for patients with an established diagnosis of ulcerative colitis, evaluation with noninvasive indices is appealing for both clinical practice and clinical trials. If the SCCAI and the Seo index can be validated, these noninvasive indices would be a low-cost way of monitoring patients in both clinical trials and in clinical practice.

\section{WHAT IS ACCEPTED AND WHAT THIS RESEARCH ADDS}

- Many disease activity indices are used for ulcerative colitis, but none have been validated.

- In clinical practice, patient-reported symptoms are often used to guide therapy without endoscopy.

- This study shows that noninvasive indices that use patientreported symptoms and blood tests correlate well with invasive indices in a wide range of disease activity in U.S. patients.

- This study shows that endoscopic items contribute little to the measurement of disease activity by standard indices.

- This study shows that the information gained from standard endoscopic items measures the same inherent factors of ulcerative colitis as patient-reported stool frequency and stool blood.

- This study shows that the use of symptoms to guide therapy of ulcerative colitis without endoscopy is justified.
- This study shows that less expensive, noninvasive indices are reasonable alternatives for measuring disease activity in clinical trials for ulcerative colitis.

\section{ACKNOWLEDGMENTS}

We would like to thank Sheryl Korsnes for her assistance in the endoscopy unit, and Jack Kalbfleisch and Brenda Gillespie for their statistical advice and assistance.

Reprint requests and correspondence: Peter D.R. Higgins, 6520 MSRB I, 1150 West Medical Center Drive, Division of Gastroenterology, University of Michigan, Ann Arbor, MI 48109.

Received September 08, 2004; accepted September 13, 2004.

\section{REFERENCES}

1. Truelove SC, Witts LJ. Cortisone in ulcerative colitis: Final report on a therapeutic trial. Br Med J 1955;1041-8.

2. Powell-Tuck J, Bown RL, Lennard-Jones JE. A comparison of oral prednisolone given as single or multiple daily doses for active proctocolitis. Scand J Gastroenterol 1978;13:8337.

3. Sutherland L, Singleton J, Sessions J, et al. Double blind, placebo controlled trial of metronidazole in Crohn's disease. Gut 1991;32:1071-5.

4. Schroeder KW, Tremaine WJ, Ilstrup DM. Coated oral 5aminosalicylic acid therapy for mildly to moderately active ulcerative colitis. A randomized study. N Engl J Med 1987;317:1625-9.

5. Seo M, Okada M, Yao T, et al. An index of disease activity in patients with ulcerative colitis. Am J Gastroenterol 1992;87:971-6.

6. Seo M, Okada M, Maeda K, et al. Correlation between endoscopic severity and the clinical activity index in ulcerative colitis. Am J Gastroenterol 1998;93:2124-9.

7. Seo M, Okada M, Yao T, et al. Evaluation of the clinical course of acute attacks in patients with ulcerative colitis through the use of an activity index. J Gastroenterol 2002;37:29-34.

8. Walmsley RS, Ayres RC, Pounder RE, et al. A simple clinical colitis activity index. Gut 1998;43:29-32.

9. Cohen RD. Outcomes in Inflammatory Bowel Disease. Clin Perspect Gastroenterol 2001;4248-51.

10. Farrell RJ. Epidermal growth factor for ulcerative colitis. $\mathrm{N}$ Engl J Med 2003;349:395-7.

11. Kim J-O, Mueller CW. Factor analysis: Statistical methods and practical issues. Sage University Papers, 1978.

12. Sinha A, Nightingale J, West KP, et al. Epidermal growth factor enemas with oral mesalamine for mild-to-moderate left-sided ulcerative colitis or proctitis. N Engl J Med 2003;349:350-7.

13. Orlandi F, Brunelli E, Feliciangeli G, et al. Observer agreement in endoscopic assessment of ulcerative colitis. Ital J Gastroenterol Hepatol 1998;30:539-41. 